\title{
ECG data compression algorithm for tele-monitoring of cardiac patients
}

\author{
Chandan Kumar Jha* and \\ Maheshkumar H. Kolekar
}

Department of Electrical Engineering, Indian Institute of Technology Patna,

Bihta - 801103, India

Email: ckjha.pee15@iitp.ac.in

Email:mahesh@iitp.ac.in

*Corresponding author

\begin{abstract}
This paper reports an efficient electrocardiogram (ECG) data compression algorithm for tele-monitoring of cardiac patients from rural area, based on combination of two encoding techniques with discrete cosine transform. The proposed technique provides good compression ratio (CR) with low percent root-mean-square difference (PRD) values. For performance evaluation of the proposed algorithm 48 records of ECG signals are taken from MIT-BIH arrhythmia database. Each record of ECG signal is of duration 1 minute and sampled at sampling frequency of $360 \mathrm{~Hz}$. Noise of the ECG signal has been removed using Savitzky-Golay filter. To transform the signal from time domain to frequency domain, discrete cosine transform has been used which compacts energy of the signal to lower order of frequency coefficients. After normalisation and rounding of transform coefficients, signals are encoded using dual encoding technique which consists of run length encoding and Huffman encoding. The dual encoding technique compresses data significantly without any loss of information. The proposed algorithm offers average values of $\mathrm{CR}, \mathrm{PRD}$, quality score, percent root mean square difference normalised, RMS error and SNR of 11.49, 3.43, 3.82, 5.51, 0.012 and $60.11 \mathrm{~dB}$ respectively.
\end{abstract}

Keywords: compression; transmission; eHealth; discrete cosine transform; Huffman encoding; run length encoding.

Reference to this paper should be made as follows: Jha, C.K. and Kolekar, M.H. (2017) 'ECG data compression algorithm for tele-monitoring of cardiac patients', Int. J. Telemedicine and Clinical Practices, Vol. 2, No. 1, pp.31-41.

Biographical notes: Chandan Kumar Jha is currently pursuing $\mathrm{PhD}$ from Indian Institute of Technology Patna in the Department of Electrical Engineering. He worked as Assistant Professor of Electronics and Communication Engineering at Institute of Information Technology and Management, Gwalior during March 2013 to December. 2014. He received his Master degree with distinction in Electronics and Communication Engineering from Birla Institute of Technology Mesra in 2012. He received his Bachelor degree in Electronics and Communication Engineering from West Bengal University of Technology Kolkata in 2010. His research interests include biomedical signal processing, ECG signal analysis and telemedicine. 
Maheshkumar H. Kolekar is working as Assistant Professor in the Department of Electrical Engineering, Indian Institute of Technology Patna since March 2010. At IIT Patna, he worked as Head of the Department, Electrical Engineering during 2013 and working as Head of the Centre for Advanced Systems Engineering since January 2014. He received his $\mathrm{PhD}$ in Electronics and Electrical Communication Engineering from Indian Institute of Technology Kharagpur in 2007. During 2008-2009, he was Post-doctoral Research Fellow in the Department of Computer Science, University of Missouri, Columbia, USA. His research interests include digital video processing, video surveillance and biomedical image and signal processing.

\section{Introduction}

Electrocardiogram is an electrophysiological signal which plays a vital role for diagnosis of heart diseases. It is a recording of electrical activity of heart muscles using electrodes placed on patient's body. For a long time of monitoring, recorded electrocardiogram (ECG) data may occupy large memory space. Compressed ECG data acquires less memory space for storage and it also helps for efficient data transmission in case of telemedicine (Dutta, 2015). Compressed ECG data also reduces time of transmission of signal as due to compression number of bits to be transmitted becomes less. Using telemedicine, expert cardiologists can receive ECG data in their own premises and after analysis of the data; they can send required medical suggestions to patient side. Thus compression of ECG data is very helpful for remote health monitoring of heart patients from rural area (Chaudhari and Karule, 2014) which is a great challenge in India. Compression algorithm of ECG data can also be helpful for big data (Sharma et al., 2014, 2015; Sharma, 2016) perspective of biomedical signal. In this paper, an efficient ECG signal compression algorithm has been proposed which offers comparatively high compression ratio and low percent root mean square difference. There are many techniques of ECG data compression. Generally these techniques can be categorised (Mamaghanian et al., 2011) as

1 direct time domain

2 transform domain

3 parameter extraction.

In direct time domain techniques, data samples of original signals are directly processed for the compression. Examples of direct data compression techniques are: turning point (TP) (Mueller, 1978), amplitude zone time epoch coding (AZTEC) (Cox et al., 1968), Fan/SAPA (Ishijima et al., 1983), coordinate reduction time encoding system (CORTES) (Abenstein and Tompkins, 1982) and entropy coding (Huffman, 1952). In transform domain method, ECG signal is converted from time domain to frequency domain and compression is performed by eliminating the insignificant spectral components. These methods include discrete cosine transform (Narasimha and Peterson, 1978), discrete wavelet transform (DWT) (Rajoub, 2002; Kolekar et al., 2013), Hermite transform (Sandryhaila et al., 2012) etc. Parameter extraction method uses combination of direct time domain and transform domain. In this method, particular features of the signal are extracted, after that compression is performed. These methods are primarily based on 
linear prediction and long-term prediction method (Nave and Cohen, 1993). These methods include feature extraction using Hilbert transform (Bolton and Westphal, 1985), curvature-based ECG signal compression (Kim et al., 2012) etc. Lai et al. (2013) proposed an algorithm which is based on normalisation, DCT-IV calculation, amplitude and sign bit separation, hybrid differential computation, non-uniform quantisation and entropy coding. The algorithm uses 64 samples in each frame and processes them one by one. Alam and Gupta (2014) proposed a differential pulse code modulation (DPCM)-based ECG coder which compresses the ECG signal without compression of QRS region. Sahoo et al. (2015) proposed an algorithm which uses empirical mode decomposition, downsampling, DCT, window filtering and Huffman encoding. Sadhukhan et al. (2015) proposed a compression algorithm based on adaptive bit encoding of DFT coefficients. Real and imaginary parts of DFT coefficients are separated and rounded, after that adaptive bit encoding is used to encode them. Mitra et al. (2012) proposed an ECG data compression technique for GSM-based offline telecardiology which was based on downsampling, single side difference generation, encoding and zero sequence compression.

This paper proposed an efficient ECG signal compression and transmission algorithm based on DCT and dual encoding technique. DCT converts ECG signal from time domain to frequency domain and it compacts energy of the signal to lower order of frequency coefficients. Using thresholding least significant coefficients are converted to zero. Normalisation and rounding operations have been performed to create many repeated data points. To improve further compression, dual encoding technique has been used which comprised of run length encoding followed by Huffman encoding. Both the encoding technique, encode data without any loss of information. This paper includes five sections. Section 2 explains the proposed compression and decompression methodology of ECG signal. Section 3 elaborates results and discussion and Section 4 includes conclusion and future scope part.

\section{Proposed methodology}

\subsection{Compression and decompression procedures}

Overview of the proposed algorithm has been shown in Figure 1 and Figure 2 which elaborate compression and decompression procedures using block diagrams. To implement the algorithm, 48 records of ECG signal have been used which are taken from MIT-BIH database (Moody and Mark, 2001). All these records have '1-channel, $360 \mathrm{~Hz}$ of sampling frequency and 11-bit resolution'. Duration of each record of the ECG signal is of 1 minute having 21,600 sample points. Before compression, each record of the ECG signal occupies $45 \mathrm{~kb}$ of memory space and after compression average space occupied by each record is $3.92 \mathrm{~kb}$. The compression procedure can be followed step by step at transmitter side of the tele-monitoring system and the decompression procedure at receiver side. The first step of the compression process is Savitzky-Golay filtering (Schafer, 2011) which smoothen the noisy ECG signal with preserving high frequency component very well. Savitzky-Golay filter of order 3 and window size of 19 has been used for filtering purpose. After filtering, downsampling has been performed to reduce the data. To transform the downsampled ECG data from time domain to frequency domain, discrete cosine transform (Narasimha and Peterson, 1978) has been used. A 
discrete cosine transform represents a finite amount of data in terms of sum of cosine functions oscillating at different frequencies. DCT (Kolekar and Sengupta, 2004) is used to concentrates energy of the signal in lower order of frequency coefficients. After implementation of DCT, thresholding operation is applied to DCT coefficients to convert least significant coefficients to zero. Normalisation with scale of 0-999 and rounding operations (Mitra et al., 2012) produce a long string of repeated data points, so it can be easily encoded by run length encoding. For normalisation and rounding operation, a normalisation constant has been defined which can be represented as

$$
\text { Normalisation Constant, } k=\frac{999}{\max (\operatorname{abs}\{\mathrm{c}(\mathrm{i})\})}
$$

where c(i) is the array of thresholded transform coefficients.

Data array generated due to normalisation and rounding operation can be represented as

$$
\mathrm{d}(\mathrm{i})=\operatorname{round}(\mathrm{k} * \mathrm{a}(\mathrm{i}))
$$

Further the normalised and rounded data array is encoded by run length encoding and Huffman encoding. Both the encoding techniques improve compression without any loss of information. To decompress ECG signal inverse Huffman encoding, inverse run length encoding, demoralisation and inverse DCT have been used. Spline interpolation (Sun et al., 2007) is used as the inverse process of down sampling. After spline interpolation, the reconstructed ECG signal is achieved.

\subsection{Run length encoding}

Run length encoding (Jha and Kolekar, 2016) has been used to encode normalised and rounded transform coefficients of ECG signal. Normalised and rounded coefficients are long runs of same data points and can be efficiently stored using run length encoding. For example

$$
X=\left[\begin{array}{lllllllllllll}
2 & 2 & 2 & 2 & 0 & 0 & 0 & 0 & 0 & 4 & 4 & 4 & 3
\end{array}\right]
$$

Can be represented using run length encoding as

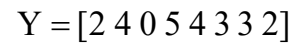

$\mathrm{X}$ contains 14 elements and encoded signal $\mathrm{Y}$ contains 8 elements. Thus run length encoding uses repetition of data and it provides improved compression performance.

\subsection{Huffman encoding}

Run length encoded data can be further compressed by Huffman encoding (Lee et al., 2011) which is a probability-based encoding technique. It is a lossless encoding technique which exploits repetition of data. In Huffman encoding, a probability-based dictionary is generated and using that dictionary data is encoded. For reconstruction of the ECG signal at the receiver side, both encoded data and dictionary must be transmitted over the communication network. Huffman encoding improves compression performance significantly. 
Figure 1 Block diagram of compression process

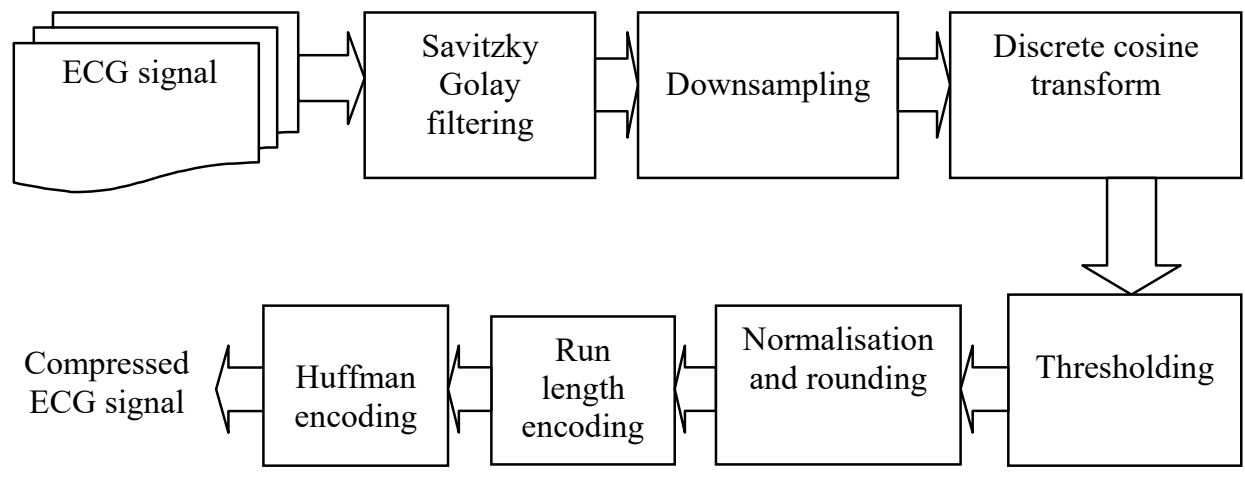

Figure 2 Block diagram of decompression process

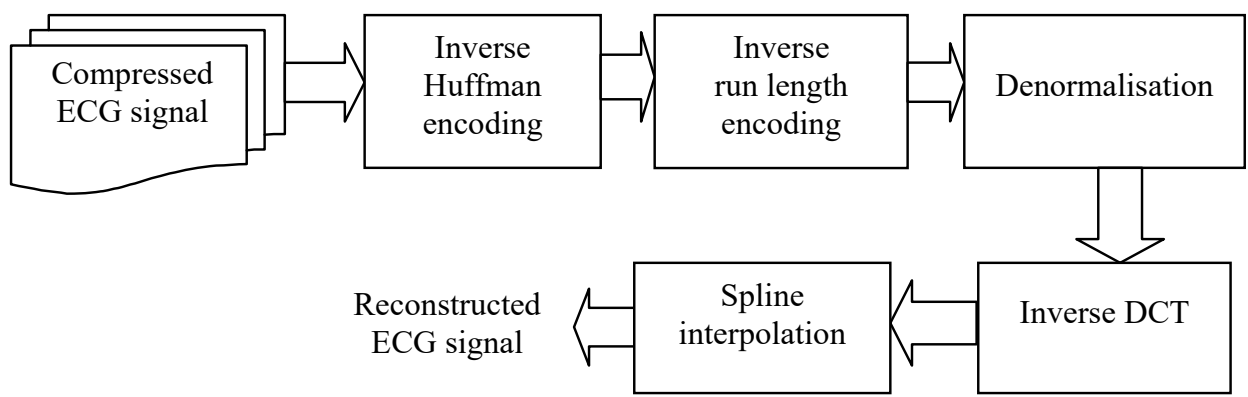

\subsection{Performance parameters}

In literature many algorithms have been used for ECG data compression. Compression performance of these algorithms can be evaluated by following performance parameters (Lee et al., 2011):

1 Compression ratio (CR):

$$
\mathrm{CR}=\frac{\text { Size of Original ECG Signal (in bytes) }}{\text { Size of Compressed ECG Signal (in bytes) }}
$$

2 Percent root-mean-square difference (PRD):

$$
\mathrm{PDR}=100 \times \sqrt{\frac{\sum_{\mathrm{n}=0}^{\mathrm{N}-1}(\mathrm{x}(\mathrm{n})-\mathrm{r}(\mathrm{n}))^{2}}{\sum_{\mathrm{n}=0}^{\mathrm{N}-1}(\mathrm{x}(n))^{2}}}
$$

where $x(n)$ is original ECG signal and $r(n)$ is reconstructed ECG signal. 
3 Quality score (QS):

$$
\mathrm{QS}=\frac{\mathrm{CR}}{\mathrm{PRD}}
$$

4 Percent root-mean-square difference normalised (PRDN):

$$
\operatorname{PRDN}=100 \times \sqrt{\frac{\sum_{n=0}^{N-1}(x(n)-r(n))^{2}}{\sum_{n=0}^{N-1}(x(n)-\overline{x(n)})^{2}}}
$$

where $\overline{\mathrm{x}(\mathrm{n})}$ is mean value of the original signal.

5 RMS error:

$$
\mathrm{RMS}=100 \times \sqrt{\frac{\sum_{\mathrm{n}=0}^{\mathrm{N}-1}(\mathrm{x}(\mathrm{n})-\mathrm{r}(\mathrm{n}))^{2}}{(\mathrm{~N}-1)}}
$$

6 Signal to noise ratio (SNR):

$$
\mathrm{SNR}=10 \times \log \left\lfloor\frac{\sum_{\mathrm{n}=0}^{\mathrm{N}-1}(\mathrm{x}(\mathrm{n})-\overline{\mathrm{x}(\mathrm{n})})^{2}}{\sum_{\mathrm{n}=0}^{\mathrm{N}-1}(\mathrm{x}(\mathrm{n})-\mathrm{r}(\mathrm{n}))^{2}}\right\rfloor
$$

\section{Results and discussion}

The compression performance of the proposed algorithm over 48 records of ECG signal has been shown in Figures 3-7. CR, PRD and QS offered by the proposed algorithm is shown in Figure 3. For a good compression algorithm $\mathrm{CR}$ value shoud be high. The maximun CR offered by the proposed algorithm is 20.46 for record no. 121 and minimum $\mathrm{CR}$ is 7.89 for record no. 208. PRD indicates the distortion of the reconstructed signal with original signal. Hence for a good compression performance PRD value should be low.The minimum PRD offered by the proposed algorithm is 1.74 for record no. 219 and it offers maximum PRD 7.20 for record no. 222. Quality score indicates reconstruction quality of the algorithm and it should be high. The proposed algorithm offers highest QS value 9.65 for record no. 121 and lowest QS value 1.66 for record no. 222. PRDN and SNR for all 48 records have been shown by Figure 4 and RMS error is shown by Figure 5. The prosposed algorithm offers average CR, PRD, QS, PRDN, RMS and SNR of $11.49,3.43,3.82,5.51,0.012$ and $60.11 \mathrm{~dB}$ respectively over 48 records of ECG signal. Figure 6 and Figure 7 shows original signal and reconstructed signal for record no. 108 and 214. From these figures, it clear that the variation of the reconstructed signal with original signal is very less. Hence the reconstructed ECG signal from the compressed ECG signal is medically accepatble. Table 1 shows comparison of the performance of the proposed algorithm in terms of CR and PRD with some existing algorithms for record no. 117 and it is clear that the proposed algorithm offers good performance. However improvement of average PRD value will result more reconstruction quality of the proposed algorithm. 
Figure 3 CR, PRD and QS for 48 records of ECG signal (see online version for colours)

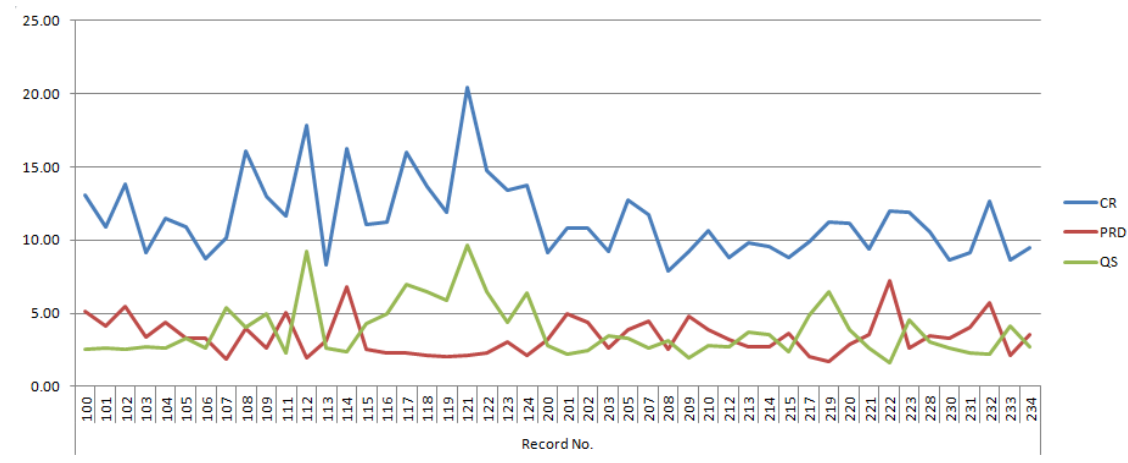

Figure 4 PRDN and SNR for 48 records of ECG signal (see online version for colours)

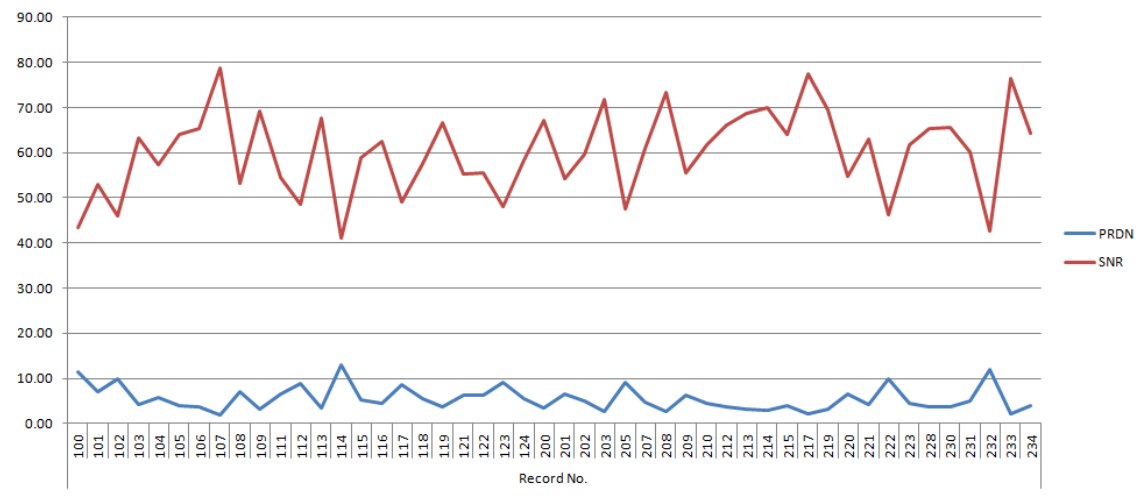

Figure 5 RMS error for 48 recordsof ECG signal (see online version for colours)

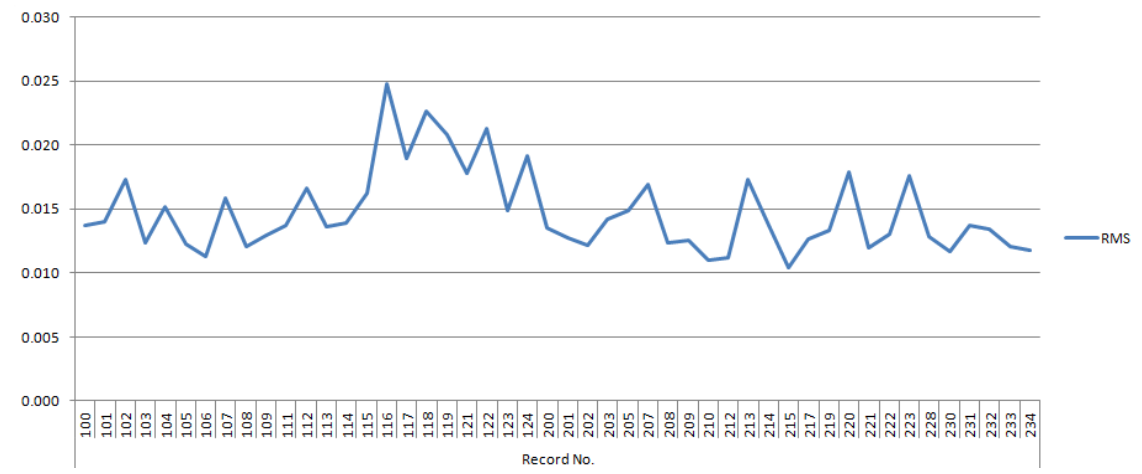


Figure 6 Original signal and reconstructed signal for record no. 108 (see online version for colours)

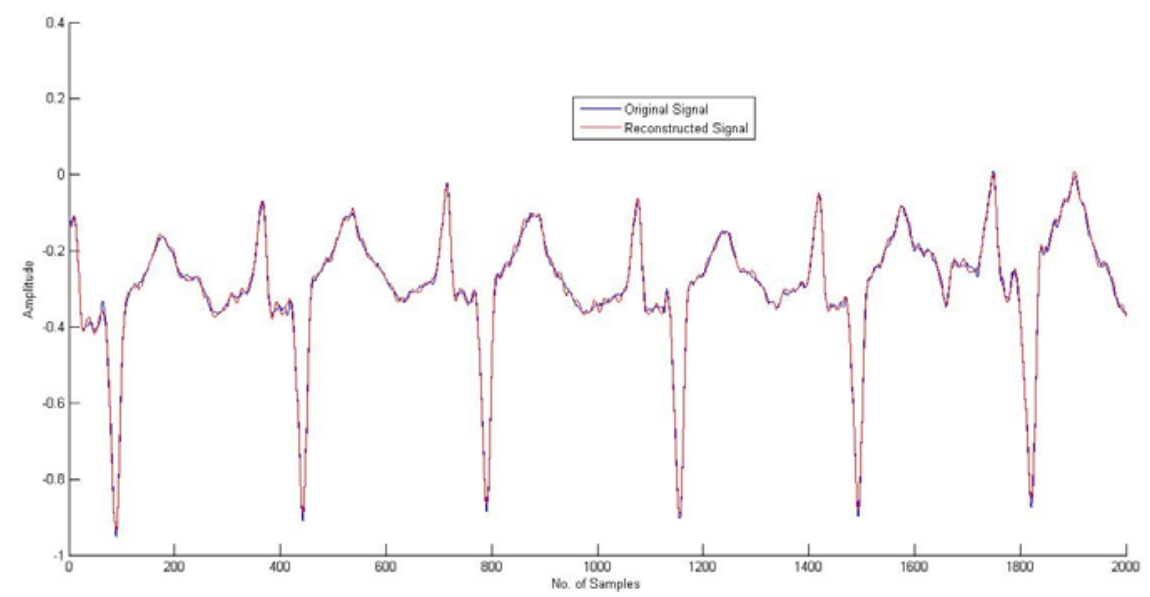

Figure 7 Original signal and reconstructed signal for record no. 214 (see online version for colours)

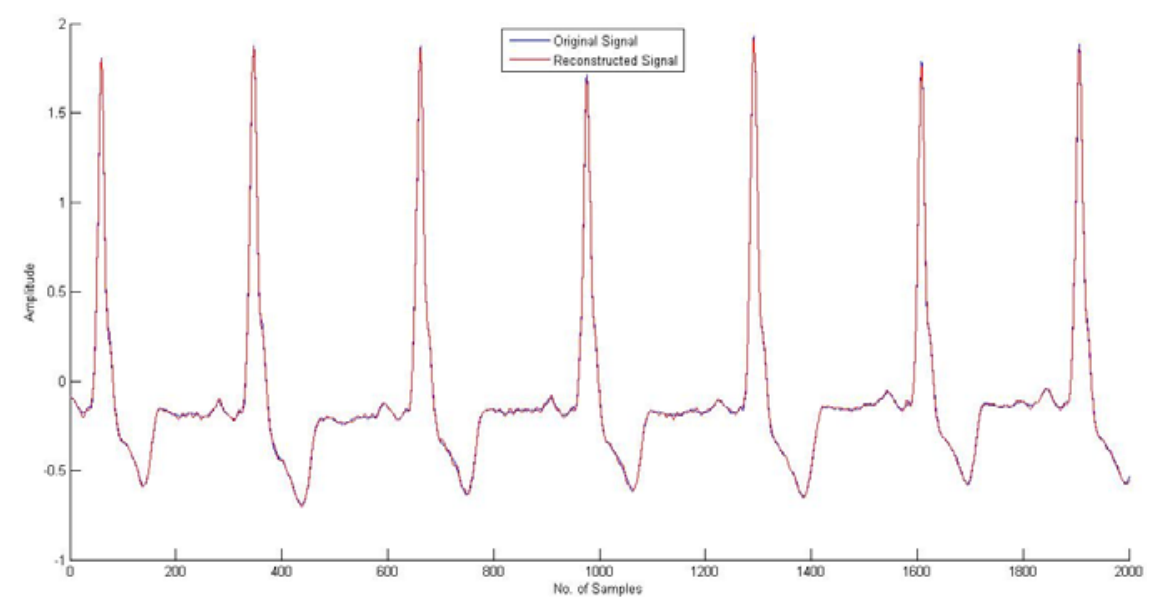

Table 1 Comparison of the compresson performance for record no. 117

\begin{tabular}{lcc}
\hline Algorithm & $C R$ & PRD (\%) \\
\hline Al-Shrouf et al. (2003) & 11.6 & 5.30 \\
Thomoglu and Sezgin (2007) & 14.9 & 5.83 \\
Lee and Lee (2008) & 7.8 & 1.00 \\
Boukhennoufa et al. (2009) & 14.3 & 2.43 \\
Hossain and Noushad (2011) & 15.1 & 2.50 \\
Kumar et al. (2013) & 5.65 & 3.63 \\
Proposed & 16.0 & 2.29 \\
\hline
\end{tabular}




\section{Conclusions and future scope}

This paper reports a DCT and dual encoding-based ECG signal compression algorithm. The proposed algorithm provides good compression performance in terms of average $\mathrm{CR}$, PRD, QS, PRDN, RMS and SNR over 48 records of ECG signals. Hence it can be used as a compression tool for all morphology of ECG signal. The proposed algorithm is suitable for ECG data compression as a powerful tool for telemedicine applications for patients suffered with cardiovascular diseases. As less PRD indicates less distortion of reconstructed signal from original signal, hence in future compression performance can be improved in terms of PRD which is average 3.43 over 48 records of ECG signal. In future we will further classify ECG data as normal data or diseased data using Bayesian network-based classifier (Kolekar and Sengupta, 2015; Kolekar, 2011) and after classification, compression algorithm can be applied to diseased ECG data for telemedicine purpose.

\section{References}

Abenstein, J.P. and Tompkins, W.J. (1982) 'A new data reduction algorithm for real time ECG analysis', IEEE Trans. Biomed. Eng., Vol. BME-29, No. 1, pp.43-48.

Alam, S. and Gupta, R. (2014) 'A DPCM based electrocardiogram coder with thresholding for real time telemonitoring applications', International Conference on Communications and Signal Processing (ICCSP), pp.176-180.

Al-Shrouf, A., Abo-Zahhad, M. and Ahmed, S.M.(2003) 'A novel compression algorithm for electrocardiogram signals based on linear prediction of the wavelet coefficients', Digital Signal Processing, Vol. 13, No. 4, pp.604-622.

Bolton, R.J. and Westphal, L.C. (1985) 'ECG display and QRS detection using the Hilbert transform', in Ripley, K.L. (Ed.): Computers in Cardiology, pp.463-466, IEEE Computer Society, Washington DC.

Boukhennoufa, N., Benmahammedm, K., Abdi, M.A. and Djeffal, F. (2009) 'Wavelet-based ECG signals compression using SPIHT technique and VKTP Coder', Int. Conf. On Signals, Circuits and Systems, ISBN: 978-1-4244-4398-7.

Chaudhari, K. and Karule, P.T. (2014) 'WiMAX network based e health service and telemedicine applications for rural and remote populations in India', International Conference on Medical Imaging, M-Health and Emerging Communication Systems, pp.398-406.

Cox, J.R., Nolle, F.M., Fozzard, H.A. and Oliver, C.G. (1968) 'AZTEC, a preprocessing program for real time ECG rhythm analysis', IEEE Trans. Biomed. Eng., Vol. BME-15, No. 4, pp. $128-129$.

Dutta, T. (2015) 'Medical data compression and transmission in wireless ad hoc networks', IEEE Sensor Journal, Vol. 15, No. 2, pp.778-786.

Hossain, M.S. and Noushad, A. (2011) 'ECG compression using subband thresholding of the wavelet coefficients', Australian Journal of Basic Applied Science, Vol. 5, No. 5, pp.739-749.

Huffman, D.A. (1952) 'A method for the construction of minimum redundancy coders', Proc. IRE, Vol. 40, No. 9, pp.1098-1101.

Ishijima, M., Shin, S.B., Hostetter, G.H. and Sklansky, J. (1983) 'Scan along polygon approximation for data compression of electrocardiograms', IEEE Trans. Biomed. Eng., Vol. BME-30, No. 11, pp.723-729.

Jha, C.K. and Kolekar, M.H. (2016) 'Efficient ECG data compression and transmission algorithm for telemedicine', 8th International Conference on Communication Systems and Networks (COMSNETS), pp.1-6. 
Kim, T-H., Kim, S-Y., Kim, J-H., Yun, B-J. and Park, K-H. (2012) 'Curvature based ECG signal compression for effective communication on WPAN', Journal of Communications and Networks, Vol. 14, No. 1, pp.21-26.

Kolekar, M.H. (2011) 'Bayesian belief network based broadcast sports video indexing', Int. Springer Journal of Multimedia Tools and Applications, Vol. 54, No. 1, pp.27-54.

Kolekar, M.H. and Sengupta, S. (2004) 'Hidden Markov model based video indexing with discrete cosine transform as a likelihood function', IEEE INDICON, pp.157-159.

Kolekar, M.H. and Sengupta, S. (2015) 'Bayesian network based customized highlight generation for broadcast soccer videos', IEEE Transaction on Broadcasting, Vol. 61, No. 2, pp.195-209.

Kolekar, M.H., Raja, G.L. and Sengupta, S. (2013) 'An introduction to wavelet based image processing and it's applications', Recent Advances in Computer Vision and Image Processing: Methodologies and Applications, IGI Global Publishers.

Kumar, R., Kumar, A. and Pandey, R.K. (2013) 'Beta wavelet based ECG signal compression using lossless encoding with modified thresholding', Computers and Electrical Engineering, Vol. 39, No. 1, pp.130-140.

Lai, S-C., Lan, C-S. and Lei, S-F. (2013) 'An efficient method of ECG signal compression by using a DCT-IV spectrum', International Conference on Communications, Circuits and Systems (ICCCAS), Vol. 1, pp.46-49.

Lee, S. and Lee, M. (2008) 'A real-time ECG data compression algorithm for a digital Holter system', 30th Annual International Conference of the IEEE Engineering in Medicine and Biology Society, pp.4736-4739.

Lee, S., Kim, J. and Lee, J.H. (2011) 'A real-time ECG data compression and transmission algorithm for an e-health device', IEEE Transactions on Biomedical Engineering, Vol. 58, No. 9, pp.2448-2455.

Mamaghanian, H., Khaled, N., Atienza, D. and Vandergheynst, P. (2011) 'Compressed sensing for real-time energy-efficient ECG compression on wireless body sensor nodes', IEEE Transactions on Biomedical Engineering, Vol. 58, No. 9, pp.2456-2466.

Mitra, M., Bera, J.N. and Gupta, R. (2012) 'Electrocardiogram compression technique for global system of mobile-based offline telecardiology application for rural clinics in India', IET Science, Measurement and Technology, Vol. 6, No. 6, pp.412-419.

Moody, G.B. and Mark, R.G. (2001) 'The impact of MIT-BIH arrhythmia database', IEEE Eng. in Med. and Biology, Vol. 20, No. 3, pp.45-50, MIT-BIH Arrhythmia Database [online] http://www.physionet.org/physiobank/database/mitdb/ (accessed 9 April 2016).

Mueller, W.C. (1978) 'Arrhythmia detection program for an ambulatory ECG monitor', Biomed. Sci. Instrument., Vol. 14, pp.81-85.

Narasimha, M. and Peterson, A. (1978) 'On the computation of the discrete cosine transform', IEEE Transactions on Communications, Vol. 26, No. 6, pp.934-93.

Nave, G. and Cohen, A. (1993) 'ECG compression using long-term prediction', IEEE Transactions on Biomedical. Engineering, Vol. 40, No. 9, pp.877-885.

Rajoub, B. (2002) 'An efficient coding algorithm for the compression of ECG signals using the wavelet transform', IEEE Transactions on Biomedical Engineering, Vol. 49, No. 4, pp.355-362.

Sadhukhan, D., Pal, S. and Mitra, M. (2015) 'Electrocardiogram data compression using adaptive bit encoding of the discrete Fourier transforms coefficients', in ET Science, Measurement and Technology, Vol. 9, No. 7, pp.866-874.

Sahoo, G.K., Ari, S. and Patra, S.K. (2015) 'Performance evaluation of ECG compression techniques', IEEE International Conference on Electrical, Computer and Communication Technologies (ICECCT), pp.1-5.

Sandryhaila, A., Saba, S., Puschel, M. and Kovacevic, J. (2012) 'Efficient compression of QRS complexes using Hermite expansion', IEEE Transactions on Signal Processing, Vol. 60, No. 2, pp.947-955. 
Schafer, R.W. (2011) 'What is a Savitzky-Golay filter?', IEEE Signal Processing Magazine, Vol. 28, No. 4, pp.111-117.

Sharma, S. (2016) 'Expanded cloud plumes hiding big data ecosystem', Future Generation Computer System, Vol. 59, pp.63-92, Elsevier.

Sharma, S., Tim, U.S., Gadia, S., Wong, J., Shandilya, R. and Peddoju, S.K. (2015) 'Classification and comparison of NoSQL big data models', International Journal of Big Data Intelligence, Vol. 2, No. 3, pp.201-221.

Sharma, S., Tim, U.S., Wong, J.S., Gadia, S. and Sharma, S. (2014) 'A brief review on leading big data models', Data Science Journal, Vol. 13, pp.138-157.

Sun, N., Ayabe, T. and Nishizaki, T. (2007) 'Efficient spline interpolation curve modeling', Third International Conference on Intelligent Information Hiding and Multimedia Signal Processing, pp.59-62.

Thomoglu, D. and Sezgin, K.E. (2007) 'ECG signal compression by multi-iteration EZW coding for different wavelets and thresholds', Computers in Biology and Medicine, Vol. 37, No. 2, pp.173-82. 